\title{
An approach to estimate the activation energies of fragmentation occurring in quadrupole collision cell of the mass spectrometer
}

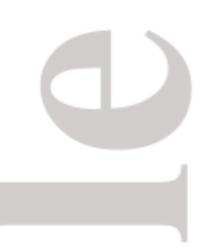

\author{
Ákos Kuki, Lajos Nagy, Miklós Zsuga, Sándor Kéki*
}

Department of Applied Chemistry, University of Debrecen, Egyetem tér 1, Debrecen H-4032, Hungary

* Corresponding author: keki.sandor@science.unideb.hu, fax: +36 52 518662; Egyetem tér 1, Debrecen H-4032, HUNGARY

\begin{abstract}
The classical semi-quantitative Rice-Ramsperger-Kassel (RRK) theory was used for the calculation of the internal energy dependent reaction rate coefficient of the collision-induced dissociation (CD) reaction in tandem mass spectrometry (MS/MS). The survival yield (SY) was determined by the reaction rate equation for the unimolecular dissociation of the precursor ion. The parameters of the rate equation and the RRK model were approximated based on the instrumental conditions. We used the RRK equation for the description of the basic behavior of the fragmentation reactions and for the estimation of the internal energy of the precursor ion. The critical energies for fragmentation $\left(E_{o}\right)$ of various molecules were estimated and compared to those reported in the literature. The model was extended by taking into account the initial internal energy distribution of the ions created in the ion source. It must be emphasized that our approach provides only a crude estimate for $E_{o}$.
\end{abstract}

Keywords: tandem mass spectrometry, collision-induced dissociation, survival yield, RRK model, collision cell

This article has been accepted for publication and undergone full peer review but has not
been through the copyediting, typesetting, pagination and proofreading process which may
lead to differences between this version and the Version of Record. Please cite this article as
doi: $10.1002 / j m s .3971$ 


\section{Introduction}

Tandem mass spectrometry (MS/MS), on the basis of collision induced dissociation (CID) fragmentation experiments, is an essential tool for elucidating the structure of ions created by soft ionization methods. In collision-induced dissociation, the mass selected precursor ion dissociates after multiple collisions with neutral gas atoms or molecules. A frequently used quantitative measure for the efficiency of the fragmentation is the survival yield (SY) defined by eq. 1 :

$$
S Y=\frac{I_{p}}{I_{p}+\sum I_{f}}
$$

where $I_{p}$ is the intensity of the precursor ion, and $\Sigma I_{f}$ is the sum of all fragment ion intensities. The survival yield analysis uses a plot of SY values as a function of the collision energy to characterize the energetics of fragmentations in collision-induced dissociation and to assess the kinetic description of the CID reactions. ${ }^{[1-8]}$ Furthermore, the SY method was also applied to make distinction between molecular ions according to their structures. ${ }^{[9-13]}$ The shape of the SY versus collision energy curves is a sigmoid type and can be well described using e.g. a four-parameter sigmoid function, as given by eq. 2 :

$$
S Y=\frac{a-b}{1+e^{\left(E_{c}-c\right) / d}}+b
$$

where $a, b, c$ and $d$ are constants and $E_{c}$ is the laboratory frame collision energy. The constants $a$ and $b$ have values close to 1 and 0 , respectively, $c$ is the collision energy necessary to obtain $50 \%$ fragmentation $\left(\mathrm{CE}_{50}\right)$, and $d$ refers to the width of the steepest part of the sigmoid curve. Eq. 2 can be used as the regression function in the fitting procedure of the experimental SY plot (determined by eq. 1). Although eq. 2 provides the characteristic collision energy $\left(\mathrm{CE}_{50}\right)$, which is an important parameter in structural analysis, using this sigmoidal function, the SY curve can purely be described mathematically.

To get deeper insight into the kinetics of the decomposition reaction in CID experiments the reaction rate coefficient $(k)$ must be determined. The rate coefficient can be expressed as a function of the internal energy $(E)$ by the classical semi-quantitative Rice-RamspergerKassel (RRK) theory (see eq. 3) or the statistical Rice-Ramsperger-Kassel-Marcus quasiequilibrium theory (RRKM/QET). ${ }^{[14]}$

$$
k(E)=v\left(1-\frac{E_{o}}{E}\right)^{s-1}
$$


where $E$ is the internal energy of the precursor ion, $v$ is the 'frequency factor', $E_{o}$ is the critical energy of the reaction, $s$ is the number of identical harmonic oscillators in the molecule that freely exchange energy (also called the number of degrees of vibrational freedom, DOF). The qualitative trends of the rate coefficient concerning the size (DOF) and the internal energy dependence can be well described by eq. 3 . Nevertheless, one of the main shortcomings of the RRK theory is the uncertainty of the number of oscillators $(s) . S$ is often referred as the number of effective oscillators, which is not equal to the total degrees of freedom. The number of oscillators is usually decreased to $20-30 \%$ of the total DOF ${ }^{[2]}$ For quantitative purpose the RRKM theory is used, ${ }^{[14]}$ which treats each oscillators independently. However, calculation of the rate coefficient by RRKM often runs into difficulties due to the lack of the reactant and transition state frequencies. Nevertheless, the classical RRK theory can also yield acceptable predictions when appropriate parameters are used. For example, the RRK framework has been successfully used for various calculations and estimations in gas phase reaction kinetics. ${ }^{[15-22]}$

In a quadrupole-type mass spectrometer the kinetic energy is transferred to the precursor ion through its multiple collisions with neutral gas molecules or atoms. ${ }^{[23]}$ In our previous study, ${ }^{[24]}$ a simple collision model was developed for the calculation of the precursor ion internal energy increase due to multiple collisions and for the computation of the time intervals between collisions. The internal energy-dependent rate coefficient $k(E)$ was calculated using both the RRK and the RRKM theory and the survival yield (SY) was determined after each collisions. One of the conclusions of our previous work was that the RRK theory proved to be capable of predicting the rate coefficient with a reasonable accuracy and can be used for estimating the critical energy for fragmentation.

In this article we simplify our multiple collision model by replacing the step by step calculation of precursor ion internal energy and reaction time $(\tau)$ with a kind of "short cut" method which uses a single $E$ and $\tau$ value in the RRK model and in rate equation, respectively. Furthermore, the RRK theory was used for the estimation of the critical energies for fragmentation $\left(E_{o}\right)$ of various molecules.

This article is protected by copyright. All rights reserved. 


\section{Experimental}

\section{Chemicals}

Leucine enkephalin, polyethylene glycol (PEG), polytetrahydrofuran (PTHF), dibenzo-18crown-6 (DB18C6), trehalose and maltose were received from Aldrich (Steinheim, Germany). Noscapine was synthesized as described in Ref [25].

\section{Electrospray Quadrupole Time-of-Flight MS/MS (ESI-Q-TOF).}

The MS/MS measurements were performed with a MicroTOF-Q type Qq-TOF MS instrument equipped with an ESI source from Bruker (Bruker Daltoniks, Bremen, Germany). The sample solutions were introduced directly into the ESI source with a syringe pump (Cole-Parmer Ins. Co., Vernon Hills, IL, USA) at a flow rate of $3 \mu \mathrm{L} / \mathrm{min}$. The temperature of the drying gas $\left(\mathrm{N}_{2}\right)$ was kept at $180^{\circ} \mathrm{C}$ and the flow rate was $4.0 \mathrm{~L} / \mathrm{min}$. The spray voltage was $4 \mathrm{kV}$. For MS/MS experiments nitrogen gas was used as the collision gas and the collision energies were varied between 8 and $100 \mathrm{eV}$ (in the laboratory frame). The pressure in the collision cell was determined to be $1.2 \times 10^{-2} \mathrm{mbar}$, except for the experiments with polytetrahydrofuran (see Fig. 4), when it was $8 \times 10^{-3} \mathrm{mbar}$ (it was determined by calibration). The precursor ions for MS/MS were selected with an isolation width of $4 \mathrm{~m} / z$. The MS/MS spectra were accumulated and recorded by a digitizer at a sampling rate of $2 \mathrm{GHz}$. The CID spectra were evaluated with the DataAnalysis 3.4 software from Bruker.

\section{Details of calculations}

The state frequencies for polyethylene glycol oligomer with eight repeat unit (PEG8) and for dibutyl phthalate were determined with standard quantum chemical calculations at B3LYP/6$31 \mathrm{G}^{*}$ level of theory.

The critical energies for fragmentation $\left(E_{o}\right)$ of various molecules reported in the literature (see Table 1) were determined: DFT method at B3LYP level with 6-31+G(d) basis set for the noscapine dimers; HF theory/6-31G(d) basis set for trehalose and maltose; B3LYP and M052X level for the lithiated PEG8; at level B3LYP/def2-TZVP for the cesiated doubly charged PEG38; and measured using threshold CID methods for the cesiated DB18C6. 


\section{Results and discussion}

\section{Describing the essential behavior of the CID reaction by the $R R K$ model}

In a CID experiment the SY value can be determined by the reaction rate of the unimolecular dissociation of the precursor ion according to eq. 4 :

$$
S Y=e^{-k \tau}
$$

where $k$ is the unimolecular decomposition rate coefficient and $\tau$ is the CID reaction time. ${ }^{[2]}$ In most of the commercially available quadrupole collision cells multiple collisions take place, the selected ions collide several times with the gas molecules that cause stepwise change in the kinetic and internal energy of the precursor ion and the average fragmentation time for an ion with a particular internal energy also changes. In this simplified approach the step by step calculation of precursor ion internal energy and reaction time was simplified by using a single $E$ and $\tau$ value in the RRK model and in rate equation, respectively. As an estimation $\tau=100 \mu \mathrm{s}$ was used which is approximately equals to the flight time in the collision cell. ${ }^{[24]}$ The uncertainty of $\tau$, e.g. the dependence on the mass of the ions, will appear in the estimated value of the 'frequency factor' ( $v$ ) in the RRK equation (eq. 3), because the product of $\tau$ and $v$ is used in the model. Both eq. 2 and 4 can be used as the regression function in the fitting procedure of the experimental SY plot (determined by eq. 1). The fitting by eq. 4 gives a deeper insight into the kinetics of the decomposition reaction in the CID experiment as the sigmoidal fit (eq. 2) and can be used for estimating the internal energy of the precursor ion or the critical energy for fragmentation $\left(E_{o}\right)$.

As the rate equation (eq. 4) suggests, the time frame of the experiment $(\tau)$ and the internal energy dependent reaction rates $(k)$ determine the observed product ion abundances in the MS/MS spectrum. The precursor ion needs more internal energy than the critical energy for fragmentation $\left(E_{o}\right)$ to produce detectable dissociation on the timescale of the tandem mass spectrometer. This excess energy is called the "kinetic shift". This is especially true for larger molecules, because the higher is the number of the oscillators there are more ways to distribute the internal energy among the vibrational degrees of freedom. As eq. 3 shows, the RRK model can reproduce this so called "DOF effect", the rate coefficient decreases rapidly with increasing number of oscillators $(s)$. Furthermore, this simple analytical expression can also predict correctly, that the dissociation rate increasing with the internal energy of the precursor ion $(E)$ and decreasing with the critical energy of fragmentation $\left(E_{o}\right)$. 
In tandem mass spectrometry, the structural analysis requires detectable abundances for the product ions and for the precursor ion as well. If the laboratory frame collision energy $\left(E_{c}\right)$ is too small dissociation hardly occurs, no product ions are generated. This lower limit can be approximated by the collision energy corresponding to $\mathrm{SY}<1 \%$. Of course, it depends on the instrumental conditions: in the case of higher sensitivity this limit can be lower. On the other hand, too high collision energy results in uninterpretable CID spectrum and/or the disappearance of the precursor ion (e.g. SY $>99 \%$ ). In the following, we discuss how the RRK formalism can predict the dissociation rate coefficient, internal energy, and lab frame collision energy necessary to achieve more than $1 \%$ and less than $99 \%$ fragmentation. First, model calculations were made keeping the critical energy for fragmentation at a constant value $E_{o}=1.2 \mathrm{eV}$ and varying the degrees of freedom of the precursor ion. Figure la shows the rate coefficient versus internal energy curves at various numbers of effective oscillators $\left(S_{\text {eff }}\right)$ calculated by eq. 3, and Fig. 1b depicts the dependence of the internal energy corresponding to the $1-99 \%$ SY range on the number of effective oscillators, respectively.

Fig. 1.

The threshold internal energies in Fig 1.b can be computed by combining eq. 3 and 4 and substituting $\mathrm{SY}=0.01$ or $\mathrm{SY}=0.99$, as follows:

$$
S Y=\exp \left(-\tau v\left(1-\frac{E_{O}}{E}\right)^{S_{e f f}}\right)
$$

and

$$
E_{0.01}=\frac{E_{O}}{1-\left(-\frac{\ln 0.01}{\tau v}\right)^{1 / S_{e f t}}} \leq E \leq \frac{E_{O}}{1-\left(-\frac{\ln 0.99}{\tau v}\right)^{1 / S_{e f t}}}=E_{0.99}
$$

where $E_{0.01}$ and $E_{0.99}$ is the internal energy corresponding to $1 \%$ and $99 \% \mathrm{SY}$, respectively, $S_{\text {eff }}$ is the number of effective oscillators. As it was already mentioned, the RRK theory overestimates the number of vibrational states, so the number of oscillators is often empirically reduced by a factor 2-5. In our previous experiments the observed SY plots could be well described by the RRK model by reducing the total degrees of freedom to about one fifth. ${ }^{[24]}$ Therefore the number of effective oscillators ( $S_{\text {eff }}$ in eq. 5 and 6) is also approximated as $0.2 \times s$ (where $s$ is the total DOF defined as $3 \times \mathrm{N}-6$, where $\mathrm{N}$ is the number of the atoms in the precursor ion). Figure 1a and b show the DOF effect unambiguously, namely that the 
internal energy needs to be increased significantly to fragment large molecules due to the distribution of internal energy over an increasing number of vibrational degrees of freedom. The fragmentation of an ion with $S_{\text {eff }}=30$ within the time window of the experiment requires internal energy in the $3.8-7.3 \mathrm{eV}$ interval, while this range is $6.9-14.0 \mathrm{eV}$ for an ion with $S_{\text {eff }}=60$ (see Fig 1a,b).

Our next aim was to determine the laboratory frame collision energy of our QTOF instrument which is necessary to achieve more than $1 \%$ and less than $99 \%$ fragmentation in the model calculations. As it was mentioned previously, in a quadrupole-type instrument the internal energy of the precursor ion and the time elapsed between the subsequent collisions are changing stepwise in the CID process. In our simplified model a constant internal energy is used, and is calculated as:

$$
E=E_{\text {init }}+\alpha E_{c}
$$

where $E_{\text {init }}$ and $E_{c}$ are the initial internal energy of the precursor ion and the laboratory frame collision energy, respectively, and $\alpha$ is the fraction of the initial kinetic energy $\left(E_{c}\right)$ converted into internal energy of the precursor ion. The initial internal energy $\left(E_{\text {init }}\right)$ was estimated as the mean thermal energy of the molecule $\left(E_{\text {therm }}\right)$ with eq. ${ }^{[2]}$

$$
E_{\text {init }}=E_{\text {therm }}=c(v, T) s k_{B} T
$$

where $s$ is the total number of oscillators (i.e. the total DOF), $k_{B}$ is the Boltzmann constant, $T$ is the absolute temperature and $c(v, T)$ is the fraction of the active oscillators, $v$ is the frequency model of the precursor ion consisting of the set of vibrational frequencies $v_{i}$, $i=1, \ldots, s$. The fraction of the active oscillators is calculated by eq. 9:[26]

$$
c(v, T)=\frac{1}{s} \sum_{i=1}^{s} \frac{h v_{i} / k T}{\exp \left(h v_{i} / k T\right)-1}
$$

$c(v, T)$ was calculated for leucine enkephalin, polyethylene glycol oligomer with eight repeat unit (PEG8), cellobiose, and dibuthyl phthalate. The state frequencies were obtained from ref. [27] for leucine enkephalin, from ref. [28] for cellobiose, and were determined by high level quantum chemical calculations for PEG8 and dibutyl phthalate (see Supporting Information Table S1 and S2). Figure S1 in the Supporting Information shows the $E_{\text {therm }} / s$ versus $T$ plots of the four compounds calculated by eq. 8 and 9. As seen in Fig. S1, the curves run 
practically together in the temperature range of 200-1000 K. Similar results were obtained for peptides by Drahos et al . ${ }^{[26]}$ The initial internal energy for ions with sufficiently large values of $s$ can be expressed as the function of the total number of oscillators, i.e. the total DOF $(s)$ by eq. 10 at the ESI-source temperature $(\sim 450 \mathrm{~K})$ :

$$
E_{\text {init }}=0.009 \times s \mathrm{eV}
$$

The $E_{\text {init }} / s$ factor in eq. 10 can be easily obtained for various temperatures from the $E_{\text {therm }} / s$ versus $T$ plots in Fig. S1. It was found, that varying this temperature by $100 \mathrm{~K}$ changed the estimated $\mathrm{E}_{\mathrm{o}}$ value by $10 \%$ in the case of leucine enkephalin.

In the following, as an approximation, eq. 10 was used for the estimation of the initial internal energies of various precursor ions.

Substituting the expression of the internal energy (eq. 7) into eq. 5 the survival yield is:

$$
S Y=\exp \left(-\tau \nu\left(1-\frac{E_{O}}{E_{\text {init }}+\alpha E_{c}}\right)^{S_{e f f}}\right)
$$

Thus the survival yield will be (by combining eq. 10 and 11):

$$
S Y=\exp \left(-\tau \nu\left(1-\frac{E_{o}}{0.009 s+\alpha E_{c}}\right)^{0.2 s}\right)
$$

where $s$ is the total DOF. It must be noted, that the $S_{\text {eff }} / s$ ratio has a large influence on the result of our model for the estimation of $E_{o}$. If $S_{\text {eff }} / s=0.3$ was used, it would decrease $E_{o}$ by $30 \%$ in the case of leucine enkephalin.

The kinetic to internal energy conversion, i.e. the value of $\alpha$ was estimated experimentally using leucine enkephalin as a "calibrant" with the critical energy for fragmentation $E_{o}=1.14 \mathrm{eV}$ proposed by Laskin. ${ }^{[29]}$ Figure 2 shows the SY versus laboratory collision energy curve for the protonated leucine enkephalin determined by the Q-TOF instrument together with the fitted curves calculated by our model with the RRK theory (eq. 12).

Fig. 2. 
The experimental SY curve was fitted to the calculated one by varying $\alpha$ and $v$ to get the best possible match. As seen in Fig. 2, excellent agreement can be achieved between the experimental and the fitted curves and $\alpha=0.32$ was obtained for the kinetic to internal energy conversion fraction, and this value will be used further here in the model calculation (eq. 12).

Fig. 3.

Figure 3 shows the variation of laboratory frame collision energy intervals to yield SY variable between $1-99 \%$ at various the numbers of effective oscillators. As Fig. 3 shows, similar trend can be seen for the laboratory frame collision energy as for the internal energy needed for $1-99 \%$ fragmentation: the interval $7.6-18.6 \mathrm{eV}$ is predicted by the RRK model for a precursor ion with $S_{\text {eff }}=30$, while the interval $13.0-35.3 \mathrm{eV}$ for an ion with $S_{\text {eff }}=60$. The dashed line in Fig. 3 represents the characteristic collision energy $\left(\mathrm{CE}_{50}\right)$ change with the number of effective oscillators. This clear linear trend coincide well with the observed linear correlation between $\mathrm{CE}_{50}$ and precursor ion mass for structurally similar molecules with nearly constant critical energy. ${ }^{[6,30]}$ Our model results a small positive intercept of this trendline $(1.95 \mathrm{eV})$, which agrees those of the trendlines of the empirical fits. ${ }^{[6]}$ Similarly, a small positive intercept can be observed in Fig. $1 \mathrm{~b}(0.62 \mathrm{eV})$. This linear increase with DOF suggests that the decreasing amount of internal energy with the increasing size (mass) is closely compensated by the increased number of collisions due to the increasing collision cross-section. From Fig. 1b and Fig. 3 it is also obvious that the internal energy and collision energy intervals are widening with the increase of the number of effective oscillators due to the less steep increase of the rate coefficient versus internal energy curve for larger $S_{\text {eff }}$ (see Fig 1a).

The RRK parameters $\tau$ and $v$ were arbitrary chosen and fixed in the model calculations (see Fig. 1 legend), but the dependence of the internal energy and the laboratory frame collision energy intervals corresponding to the $1-99 \%$ SY on the $\tau \times v$ product was also estimated. A three order of magnitude increase of the $\tau \times v$ product decreased the internal energy and the laboratory frame collision energy intervals to about one half and one fourth, respectively.

In order to justify the prediction capability of our model (eq. 12), we compared the calculated and observed SY values of our former experiments published in ref. [30].

This article is protected by copyright. All rights reserved. 
Fig. 4.

Figure 4a shows the survival yield versus collision energy curves for doubly charged polytetrahydrofuran (PTHF) with number of repeat units of 8,13 , and 19, corresponding to

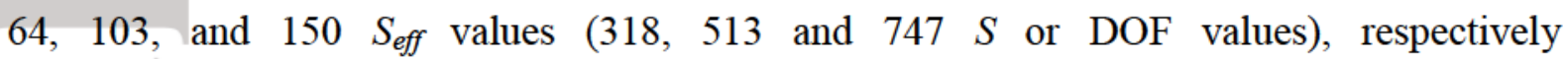
$\left(S_{\text {eff }}=\mathrm{DOF} / 5\right)$. The solid lines represent the calculated SY curves by our RRK model (eq. 12) with the parameters $\tau=100 \mu$ s and $\alpha=0.2$. (The latter is smaller than the $\alpha=0.32$ value mentioned above as model parameter and used in the following in this paper, because these experiments were performed at different instrumental conditions due to the collision cell replacement.) The parameters $E_{o}$ and $v$ in eq. 12 were determined by fitting the calculated data to the experimental SY versus collision energy curve. ( $E_{o}$ and $v$ were varied using a spreadsheet software until the best fit to the experimental SY values was obtained.) As seen in Fig 4a, our simplified RRK model can treat the DOF effect properly, good fits could be achieved for all the three curves using the same $E_{o}$ and $v$ values $2.9 \mathrm{eV}$ and $3.0 \times 10^{9} \mathrm{~s}^{-1}$ respectively. To achieve perfect match between the experimental and calculated curves only a slight modification of the $\alpha$ values was necessary for the PTHFs with different number of repeat units: $\alpha=0.206, \alpha=0.195, \alpha=0.204$, for PTHF with 8,13 , and 19 repeat unit, respectively. The calculated collision energy intervals corresponding to the $1-99 \%$ SY range show also good agreement with the measure ones as it can be seen in Fig $4 \mathrm{~b}$.

As the RRK equation (eq. 12) suggests, besides the DOF or $S_{\text {eff }}$ of the precursor ion, the critical energy for fragmentation $\left(E_{o}\right)$ also determines the dissociation rate coefficient, and the internal energy and collision energy intervals necessary for structural analysis. Keeping the number of effective oscillators at a constant value of $S_{\text {eff }}=50$ and varying the critical energy for fragmentation of the precursor ion similar model calculations were made and figures were constructed as in the previous section (see Fig. 5).

Fig. 5.

The predictions of the RRK model are analogous to those when the $S_{\text {eff }}$ was varied, the same trends can be seen in Fig. 1 and Fig. 5. The increase of $S_{\text {eff }}$ and $E_{o}$ both shift the internal energy and lab frame collision energy intervals corresponding to the $1-99 \%$ SY range into higher energies, and widen them. Our model formally results zero intercept for the trendline 
of the points corresponding to $50 \% \mathrm{SY}$ in Fig. $5 \mathrm{~b}$, and $-E_{\text {init }} / \alpha(-7.03 \mathrm{eV}$ in the model calculations) intercept for the similar trendline in Fig. 5c. The negative collision energy values at $E_{o}=0.3$ indicate that with low critical energy the ions may dissociate before they enter the collision cell.

\section{Estimation of the critical energy for fragmentation $\left(E_{o}\right)$ using the RRK model}

Our next aim was to estimate the critical energies for fragmentation of various molecules and compare our results with those found in the literature to check the validity of our "short cut" model. It must be noted, that very few accurate $E_{0}$ values are reported in the literature. As an example, Fig. 6 shows the SY versus laboratory frame collision energy curve for the sodiated noscapine dimer $\left([2 \mathrm{M}+\mathrm{Na}]^{+}\right)$determined by our Q-TOF instrument together with the fitted curve calculated by the RRK model (eq. 12).

Fig. 6.

The experimental SY curve was fitted to the calculated one varying $E_{o}$ and $v$ to get the best possible match. As seen in Fig. 6, excellent agreement can be achieved between the experimental and the fitted curves. The estimated $E_{o}=0.80 \mathrm{eV}$ value is very close to that determined by quantum chemical calculations. ${ }^{[13]}$ The estimated critical energies for fragmentation are compiled in Table 1. As the values in Table 1 show, despite the simplifications and approximations of the RRK model it may be still capable of the rough estimation of the critical energies for fragmentation. The uncertainty of the calculated critical energy was estimated by the Gaussian error propagation rule. The supposed errors of the three parameters in eq. 12 are as follows, initial internal energy ( $\left.\mathrm{E}_{\text {init }}\right): 20 \%$, the $S_{\text {eff }} / s$ ratio: $25 \%$, and $\alpha: 17 \%$. The partial derivates in the Gaussian error propagation formula was determined by multiple fittings varying one of the three parameters. $21 \%$ relative error was obtained. But we have to take into consideration the limitations and assumptions of the RRK theory itself, thus the accuracy of our method is estimated to be $30 \%$, and this value was used in Table 1 as the uncertainty.

Table 1. 


\section{Effect of the initial internal energy distribution on the SY curves}

Our model was extended by taking into account the initial internal energy distribution of the ions created in the ion source. The internal energy distribution $P(E)$ is calculated by eq. 13 : $^{\text {[26] }}$

$$
P(E)=\frac{\rho(E) \exp (-E / k T)}{\int_{0}^{\infty} \rho(E) \exp (-E / k T)}
$$

where $\rho(E)$ is the density of states at energy $E$. The density of states was calculated by the Beyer-Swinehart direct count method ${ }^{[36]}$ using a homemade software. The calculations were made for two compounds: protonated dibutyl phthalate and protonated leucine enkephalin. The state frequencies necessary for the direct count method were obtained from ref. [27] for leucine enkephalin and were determined by high level quantum chemical calculations for dibutyl phthalate.

Using the initial internal energy distribution instead of an average initial internal energy can

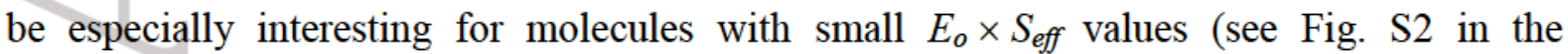
Supporting Information), because in this case the ions with higher initial internal energy may dissociate before they enter the collision cell. Concerning the survival yield calculation the path of the ions can be divided into three regions in a commonly used Q-TOF machine. (i) The decomposition from the ion source to the first analyzer of the tandem mass spectrometer (i.e. the quadrupole mass filter in our Q-TOF instrument) alters the original internal energy distribution by decreasing the probability of the higher internal energies. We estimated this distortion of the internal energy distribution for dibutyl phthalate as a model compound using the RRK model for the calculation of the SY values prior the first quadrupole. The time frame was estimated to be $100 \mu$ s for this region. It was found, that only a slight decrease of the higher tail of the internal energy distribution function can be expected, as seen in Fig. S2 in the Supporting Information. Pak et al. calculated the internal energy distributions of the precursor ion at different points of the mass spectrometer and found similar decrease of the higher tail prior the collision cell (Fig 2.b in ref. [23]). (ii) The decomposition in the first analytical quadrupole might also influence the observed SY, but this may be neglected, because the time frame for the dissociation probably even smaller here than in the first region (from the ion source to the quadrupole). (iii) The SY values at the end of the collision cell were calculated according to eq. 14:

This article is protected by copyright. All rights reserved. 


$$
S Y=\int_{0}^{\infty} P(E) e^{-k(E) \tau} d E
$$

where $P(E)$ is the internal energy distribution, $k$ is the decomposition reaction rate coefficient and $\tau$ is the CID reaction time.

For comparison, the SY values were calculated using a single internal energy value (by eq. 12) and by eq. 14 , providing thermal distribution. In both cases, the parameters $E_{o}$ and $v$ (latter identical for the single and distribution case) in eq. 12 were determined by fitting the calculated data to the experimental SY versus collision energy curve. As Fig. S3 in the Supporting Information shows, for the protonated dibutyl phthalate good fit could be achieved for both calculations using the $E_{o}$ values $0.26 \mathrm{eV}$ and $0.27 \mathrm{eV}$, in the case of a single internal energy value and internal energy distribution, respectively. The same calculations were performed for leucine enkephalin. As it was expected, even smaller difference was obtained between the fitting parameters using single internal energy $\left(E_{o}=1.14 \mathrm{eV}\right)$ or internal energy distribution $\left(E_{o}=1.15 \mathrm{eV}\right)$ in the calculations.

\section{Comparison of the efficiency of fragmentation on different quadrupole type mass spectrometers}

Although this is not the main goal of this paper, it may be interesting to see, what the reasons are for the differences between the SY plots (namely the efficiency of fragmentation) recorded on different instruments. The SY plot of leucine enkephalin presented in Fig. 2 was compared to the one measured on a Waters QQQ instrument by Bazsó et al. (Figure 6 in ref. [37], it was digitalized and presented as Fig. S4 in the Supporting Information). The characteristic point of the SY plot belongs to $50 \%$ fragmentation efficiency ( $S Y=0.5$ ), the abscissa of this point is the characteristic collision energy $\left(\mathrm{CE}_{50}\right)$. Substituting $\mathrm{SY}=0.5$ into our model (eq. 11), the characteristic collision energy can be expressed as:

$$
C E_{50}=\left(\frac{S_{\text {eff }} E_{o}}{\ln (\tau \nu)-\ln (\ln 2)}-E_{\text {init }}\right) / \alpha
$$

The detailed derivation of eq. 15 can be found in the Supporting Information. As eq. 15 shows, for the same molecule (same $S_{\text {eff }}, E_{o}$, and $v$ ) the efficiency of the fragmentation depends on the instrumental parameters: (i) initial internal energy of the precursor ion $\left(E_{\text {init }}\right)$, which is basically determined by the source conditions, (ii) the time frame of the CID 
reaction $(\tau)$, (iii) and the fraction of the initial kinetic energy converted into internal energy of the precursor ion $(\alpha)$. Accordingly, the experimental SY curve recorded on the QQQ instrument was fitted to the calculated SY curve (eq. 11) varying $\tau$ and $\alpha$ to get the best possible match (see Fig. S4 in the Supporting Information). The third instrumental parameter, $E_{\text {init, }}$ was estimated with eq. 8 based on the source conditions of the QQQ instrument. The same $S_{\text {eff }}, E_{o}$, and $v$ were used as were obtained previously (see Fig. 2). The estimated $\tau=161 \mu$ s and $\alpha=0.39$ values express the difference between the conditions of the two different instrument (the QTOF values were: $\tau=100 \mu$ s and $\alpha=0.32$ ). The $\mathrm{CE}_{50}$ values determined by the fitted functions were $17.3 \mathrm{eV}$ and $21.0 \mathrm{eV}$ for the QQQ and QTOF instrument, respectively. The lower laboratory frame collision energy necessary to achieve $50 \%$ fragmentation in the case of the QQQ instrument is due to the longer CID reaction time $(\tau)$, and the larger $\alpha$ factor, as our model suggests.

\section{Conclusions}

Using the RRK model for the calculation of the internal energy dependent rate coefficient in the kinetic equation of the $\mathrm{CID}$ reactions, the basic behavior of the fragmentation reactions can be described. On the basis of previous experiments approximations were used to simplify the calculations. These approximations, which depend on the experimental conditions were the following: (i) the number of effective oscillators $S_{\text {eff }}=\mathrm{DOF} / 5$ was used, and this approximation can be used for ions with sufficiently large values of DOF, (ii) the fraction of $\alpha=0.32$ of the laboratory frame collision energy was converted into internal energy in the collision cell and (iii) the estimate $100 \mu$ s was used as the CID reaction time. It must be emphasized that the value of $\alpha$ strongly depends on the instrumental parameters: $\alpha=0.2$ was used to process the data recorded with different collision cell conditions and $\alpha=0.39$ was determined for a QQQ instrument ${ }^{37}$. After estimating the kinetic to internal energy conversion (the value of $\alpha$ ), e.g. using leucine enkephalin as a "calibrant", our simplified so called "short cut" method is capable to estimate the internal energy and the laboratory frame collision energy intervals necessary for the MS/MS structural analysis of the precursor ion, which has both theoretical and practical importance. Not only the qualitative trends concerning the size (DOF) and critical energy dependence of the CID reaction can be predicted by the RRK model, but estimates can also be given for the critical energy for 
fragmentation $\left(E_{o}\right)$. In this study our aim was only to give a rough estimation on the $E_{o}$ values, using $v$ only as a fitting parameter not a goal value, because the product $\tau \times v$ was used in the fitting procedure so the uncertainties in the $\tau$ values hinder the estimation of $v$. In addition, using the RRK model the variation of $S_{\text {eff }}$ with the internal energy was also

neglected resulting inaccurate $v$ values. ${ }^{[24]}$ The relative error of the calculated $E_{o}$ values originated from the approximations and from the uncertainties of the model parameters (initial internal energy, number of effective oscillators, kinetic to internal energy conversion ratio) was evaluated to be $30 \%$. Our model calculations for dibutyl phthalate and leucine enkephalin suggest, that the approximation of using a single initial internal energy besides the initial internal energy distribution of the precursor ion can be tolerated.

Due to its limitations and assumptions the semi-quantitative RRK theory is basically helpful for qualitative purposes. Our method provides a simple but only a rough description of the energetics of fragmentations in collision induced dissociation (CID) and capable for only the crude estimation of the critical energy for fragmentation $\left(E_{o}\right)$.

\section{Acknowledgment}

The work was supported by the GINOP-2.3.2-15-2016-00041 project. The project was cofinanced by the European Union and the European Regonal Development Fund. Furthermore, this paper was also supported by the János Bolyai Research Scholarship of the Hungarian Academy of Sciences and by the grant K-116465, and supported through the New National Excellence Program of the Ministry of Human Capacities, ÚNKP-16-3 (T. Nagy).

We acknowledge Mihály Purgel (Department of Physical Chemistry, University of Debrecen) for his help in quantum chemical calculations

\section{References}

[1] F. Derwa, E.D. Pauw, P. Natalis. New basis for a method for the estimation of secondary ion internal energy-distribution in soft ionization techniques. Org. Mass Spectrom. 1991, 26, 117.

[2] K. Vékey. Internal energy effects in mass spectrometry. J. Mass Spectrom. 1996, 31, 445 .

[3] P.B. Armentrout. Threshold collision-induced dissociations for the determination of accurate gas-phase binding energies and reaction barriers. in Modern Mass Spectrometry, (Ed: C.A. Schalley), Springer-Verlag, Berlin, 2003, pp. 233-262.

This article is protected by copyright. All rights reserved. 
[4] X.H. Guo, M.C. Duursma, P.G. Kistemaker, N.M.M. Nibbering, K. Vekey, L. Drahos, R.M. Heeren. Manipulating internal energy of protonated biomolecules in electrospray ionization Fourier transform ion cyclotron resonance mass spectrometry. J. Mass Spectrom. 2003, 38, 597.

[5] V. Gabelica, E. De Pauw. Internal energy and fragmentations of ions produced in electrospray sources. Mass Spectrometry Rev. 2005, 24, 566.

[6] A. Memboeuf, A. Nasioudis, S. Indelicato, F. Pollreisz, Á. Kuki, S. Kéki, O.F. van den Brink, K. Vékey, L. Drahos. Size effect on fragmentation in tandem mass spectrometry. Anal. Chem. 2010, 82, 2294.

[7] L. Drahos, R.M.A. Heeren, C. Collette, E. De Pauw, K. Vékey. Thermal energy distribution observed in electrospray ionization. J. Mass Spectrom. 1999, 34, 1373.

[8] J. Naban-Maillet, D. Lesage, A. Bossée, Y. Gimbert, J. Sztáray, K. Vékey, J-C. Tabet. Internal energy distribution in electrospray ionization. J. Mass Spectrom. 2005, 40, 1.

[9] I. Haller, U.A. Mirza, B.T. Chait. Collision induced decomposition of peptides. Choice of collision parameters. J. Am. Soc. Mass Spectrom. 1996, 7, 677.

[10] T.M. Kertesz, L.H. Hall, D.W. Hill, D.F. Grant. CE50: quantifying collision induced dissociation energy for small molecule characterization and identification. J. Am. Soc. Mass Spectrom. 2009, 20, 1759.

[11] A. Nasidoudis, A. Memboeuf, R.M.A. Heeren, D.F. Smith, K. Vekey, L. Drahos, O.F. van den Brink. Discrimination of polymers by using their characteristic collision energy in tandem mass spectrometry. Anal. Chem. 2010, 82, 9350.

[12] A. Memboeuf, L. Jullien, R. Lartia, B. Brasme, Y. Gimbert. Tandem mass spectrometric analysis of a mixture of isobars using the survival yield technique. J. Am. Soc. Mass Spectrom. 2011, 22, 1744.

[13] T. Nagy, Á. Kuki, B. Antal, L. Nagy, M. Purgel, A. Sipos, M. Nagy, M. Zsuga, S. Kéki. Chiral differentiation of the noscapine and hydrastine stereoisomers by electrospray ionization tandem mass spectrometry. J. Mass Spectrom. 2015, 50, 240.

[14] T. Baer, P.M. Mayer. Statistical Rice-Ramsperger-Kassel-Marcus quasiequilibrium theory calculations in mass spectrometry. J. Am. Soc. Mass Spectrom. 1997, 8, 103. 
[15] G. Chen, R.G. Cooks, D.M. Bunk, M.J. Welch, J.R. Christie. Partitioning of kinetic energy to internal energy in the low energy collision-induced dissociations of protonbound dimers of polypeptides. Int. J. Mass Spectrom. 1999, 185/186/187, 75.

[16] T. Hanmura, M. Ichihashi, Y. Watanabe, N. Isomura, T. Kondow. Reactions of Nitrogen Monoxide on Cobalt Cluster Ions: Reaction Enhancement by Introduction of Hydrogen. J. Phys. Chem. A 2007, 111, 422.

[17] S. Nordholm, A. Back, A.: On the role of nonergodicity and slow IVR in unimolecular reaction rate theory - A review and a view. Phys. Chem. Chem. Phys. 2001, 3, 2289.

[18] B.B. Schneider, D.J. Douglas, D.D.Y. Chen. Collision-induced dissociation of bradykinin ions in the interface region of an ESI-MS. J. Am. Soc. Mass Spectrom. 2001, 12,772 .

[19] R. Jansen, I. Wysong, S. Gimelshein, M. Zeifman, U. Buck. Nonequilibrium numerical model of homogeneous condensation in argon and water vapor expansions. J. Chem. Phys. 2010, 132, 244105.

[20] J. Hagen, L.D. Socaciu, U. Heiz, T.M. Bernhardt, L. Woste. Size dependent reaction kinetics of small gold clusters with carbon monoxide: Influence of internal degrees of freedom and carbonyl complex stability. Eur. Phys. J. D 2003, 24, 327.

[21] E. Segev, T. Wyttenbach, M.T. Bowers, R.B. Gerber. Conformational evolution of ubiquitin ions in electrospray mass spectrometry: molecular dynamics simulations at gradually increasing temperatures. Phys. Chem. Chem. Phys. 2008, 10, 3077.

[22] K.M. Ervin. Microcanonical analysis of the kinetic method. The meaning of the “effective temperature". Int. J. Mass Spectrom. 2000, 195/196, 271.

[23] A. Pak, D. Lesage, Y. Gimbert, K. Vékey, J-C. Tabet. Internal energy distribution of peptides in electrospray ionization : ESI and collision-induced dissociation spectra calculation. J. Mass Spectrom. 2008, 43, 447.

[24] Á. Kuki, G. Shemirani, L. Nagy, B. Antal, M. Zsuga, S. Kéki. Estimation of activation energy from the survival yields: fragmentation study of leucine enkephalin and polyethers by tandem mass spectrometry. J. Am. Soc. Mass Spectrom. 2013, 24, 1064.

[25] Gy. Gaál, P. Kerekes, R. Bognár. About the accompanying alkaloids of morphine VI. Preparation of the narcotine isomers. J. Praktisch. Chem. 1971, 313, 935. 
[26] L. Drahos, K. Vékey. Determination of the thermal energy and its distribution in peptides. J. Am. Soc. Mass Spectrom. 1999, 10, 323.

[27] J. Sztáray, A. Memboeuf, L. Drahos, K. Vékey. Leucine enkephalin - a mass spectrometry standard. Mass Spectrom. Rev. 2011, 30, 298.

[28] V.M. Andrianov, R.G. Zhbankov, V.G. Dashevskii. A theoretical study of the vibrational spectrum of cellobiose within the framework of the additive model of interatomic interactions. J. Struct. Chem. 1980, 21, 320.

[29] J. Laskin. Energetics and dynamics of fragmentation of protonated leucine enkephalin from time- and energy-resolved surface-induced dissociation studies. J. Phys. Chem. A 2006, 110,8554 .

[30] Á. Kuki, L. Nagy, A. Memboeuf, L. Drahos, K. Vékey, M. Zsuga, S. Kéki. Energydependent collision-induced dissociation of lithiated polytetrahydrofuran: effect of the size on the fragmentation properties. J. Am. Soc. Mass Spectrom. 2010, 21, 1753.

[31] T. Yamagaki, K. Fukui, K. Tachibana. Analysis of glycosyl bond cleavage and related isotope effects in collision-induced dissociation quadrupole-time-of-flight mass spectrometry of isomeric trehaloses. Anal. Chem. 2006, 78, 1015.

[32] H. Suzuki, A. Kameyama, K. Tachibana, H. Narimatsu, K. Fukui. Computationally and experimentally derived general rules for fragmentation of various glycosyl bonds in sodium adduct oligosaccharides. Anal. Chem. 2009, 81, 1108.

[33] A. Memboeuf, L. Drahos, K. Vékey, G. Lendvay. Energetics of fragmentation for cationized poly(ethylene glycol) oligomers. Rapid Comm. Mass Spectrom. 2010, 24, 2471.

[34] A. Memboeuf, K. Vekey, G. Lendvay. Structure and energetics of poly(ethylene glycol) cationized by $\mathrm{Li}+, \mathrm{Na}+, \mathrm{K}+$ and $\mathrm{Cs}+$ : a first-principles study. Eur. J. Mass Spectrom. 2011, 17, 33 .

[35] J.D. Anderson, E.S. Paulsen, D.V. Dearden. Alkali metal binding energies of dibenzo18-crown-6: experimental and computational results. Int. J. Mass Spectrom. 2003, 227, 63.

[36] T. Beyer, D.F. Swinehart. Number of multiply-restricted partitions [A1] (Algorithm 448). Commun. ACM, 1973, 16, 379. 
[37] F.L. Bazsó, O. Ozohanics, G. Schlosser, K. Ludányi, K. Vékey, L. Drahos. Quantitative comparison of tandem mass spectra obtained on various instruments. J. Am. Soc. Mass Spectrom. 2016, 27, 1357.

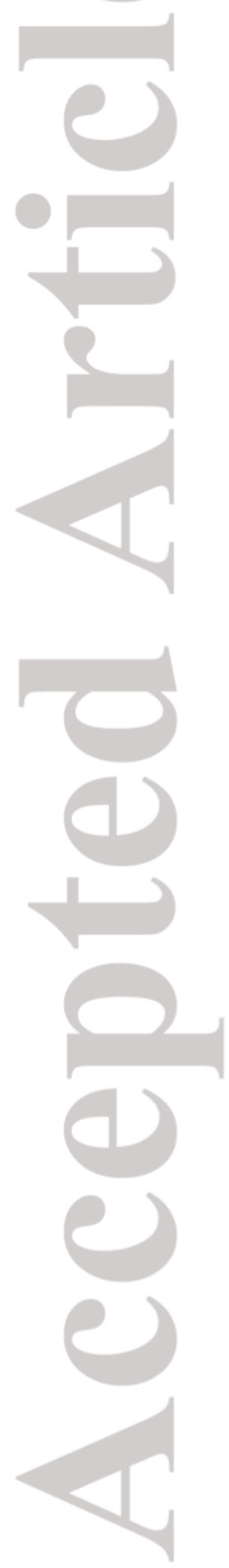


Table 1. Critical energy for fragmentation values of different ions, estimated by the RRK equation based on the CID experiments on the QTOF instrument, and literature values.

\begin{tabular}{|c|c|c|c|c|}
\hline \multirow{2}{*}{ Precursor ion } & \multirow{2}{*}{$S(\mathrm{DOF})$} & \multicolumn{2}{|c|}{ Critical energy; $E_{o}(\mathrm{eV})$} & \multirow{2}{*}{ Ref. } \\
\hline & & Calc. by RRK ${ }^{a}$ & Literature $^{\mathrm{b}}$ & \\
\hline noscapine dimer $[2 \mathrm{M}+\mathrm{Na}]^{+}$ & 309 & $0.80 \pm 0.24$ & 0.75 & [13] \\
\hline noscapine dimer $[2 \mathrm{M}+\mathrm{K}]^{+}$ & 309 & $0.43 \pm 0.13$ & 0.36 & [13] \\
\hline noscapine dimer $[2 \mathrm{M}+\mathrm{Cs}]^{+}$ & 309 & $0.35 \pm 0.11$ & 0.29 & {$[13]$} \\
\hline trehalose $[\mathrm{M}+\mathrm{Na}]^{+}$ & 132 & $1.72 \pm 0.52$ & 1.69 & {$[31]$} \\
\hline maltose $[\mathrm{M}+\mathrm{Na}]^{+}$ & 132 & $2.27 \pm 0.68$ & 2.66 & {$[32]$} \\
\hline lithiated PEG8 ${ }^{\mathrm{c}}$ & 174 & $3.1 \pm 0.93$ & $2.3-2.8$ & {$[33]$} \\
\hline cesiated doubly charged PEG38 ${ }^{d}$ & 807 & $2.87 \pm 0.86$ & $3.27 \pm 0.22$ & {$[34]$} \\
\hline cesiated DB18C6 $6^{\mathrm{e}}$ & 147 & $1.07 \pm 0.32$ & $1.42 \pm 0.36$ & {$[35]$} \\
\hline
\end{tabular}

${ }^{\mathrm{a}}$ The estimated error of $E_{o}$ is indicated as \pm values

${ }^{\mathrm{b}}$ The calculation methods are detailed in the Experimental section.

polyethylene glycol, $\left[\mathrm{HO}\left(\mathrm{C}_{2} \mathrm{H}_{4} \mathrm{O}\right)_{8} \mathrm{H}+\mathrm{Li}\right]^{+}$

d polyethylene glycol, $\left[\mathrm{HO}\left(\mathrm{C}_{2} \mathrm{H}_{4} \mathrm{O}\right)_{38} \mathrm{H}+2 \mathrm{Cs}\right]^{2+}$; product ions: $\left[\mathrm{HO}\left(\mathrm{C}_{2} \mathrm{H}_{4} \mathrm{O}\right)_{38} \mathrm{H}+\mathrm{Cs}\right]^{+}$and $\mathrm{Cs}^{+}$

dibenzo-18-crown-6 
Figure 1.
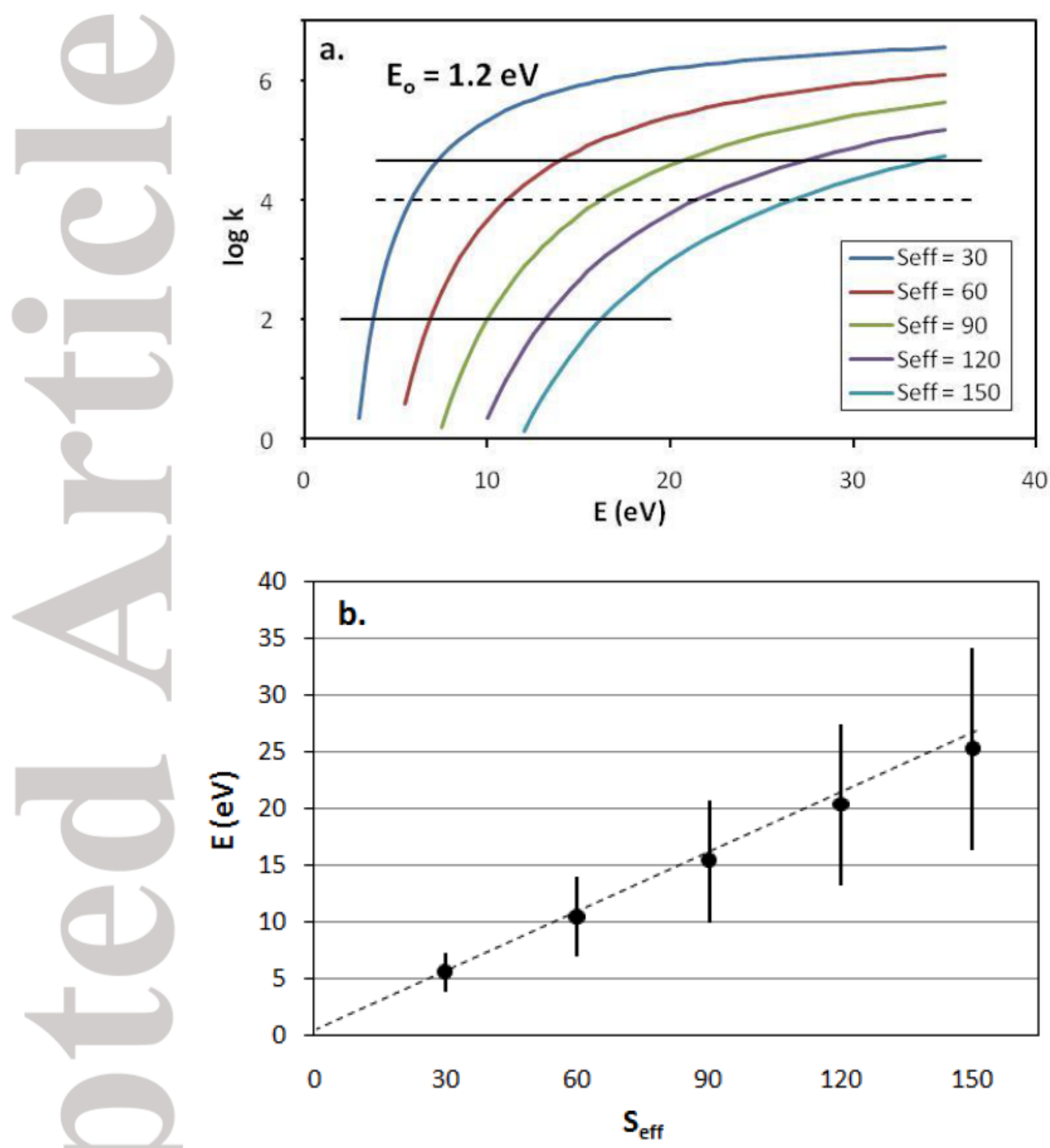

Fig. 1.

(a.) The dissociation rate coefficient $(k)$ versus internal energy $(E)$ curves calculated by the RRK equation at different number of effective oscillators $\left(S_{\text {eff }}\right)$. The horizontal lines represent the rate coefficient intervals corresponding to the $1-99 \%$ SY range. (b.) The vertical bars represent the variation of internal energy intervals to yield SY variable between $1-99 \%$. The dashed lines correspond to the $k \tau=1$ value. The black points indicate the internal energy corresponding to $50 \% \mathrm{SY}\left(\mathrm{CE}_{50}\right)$. RRK parameters were arbitrary chosen in the model calculations: $\tau=100 \mu \mathrm{s}, \nu=10^{7} \mathrm{~s}^{-1}, E_{o}=1.2 \mathrm{eV}$. 
Figure 2.

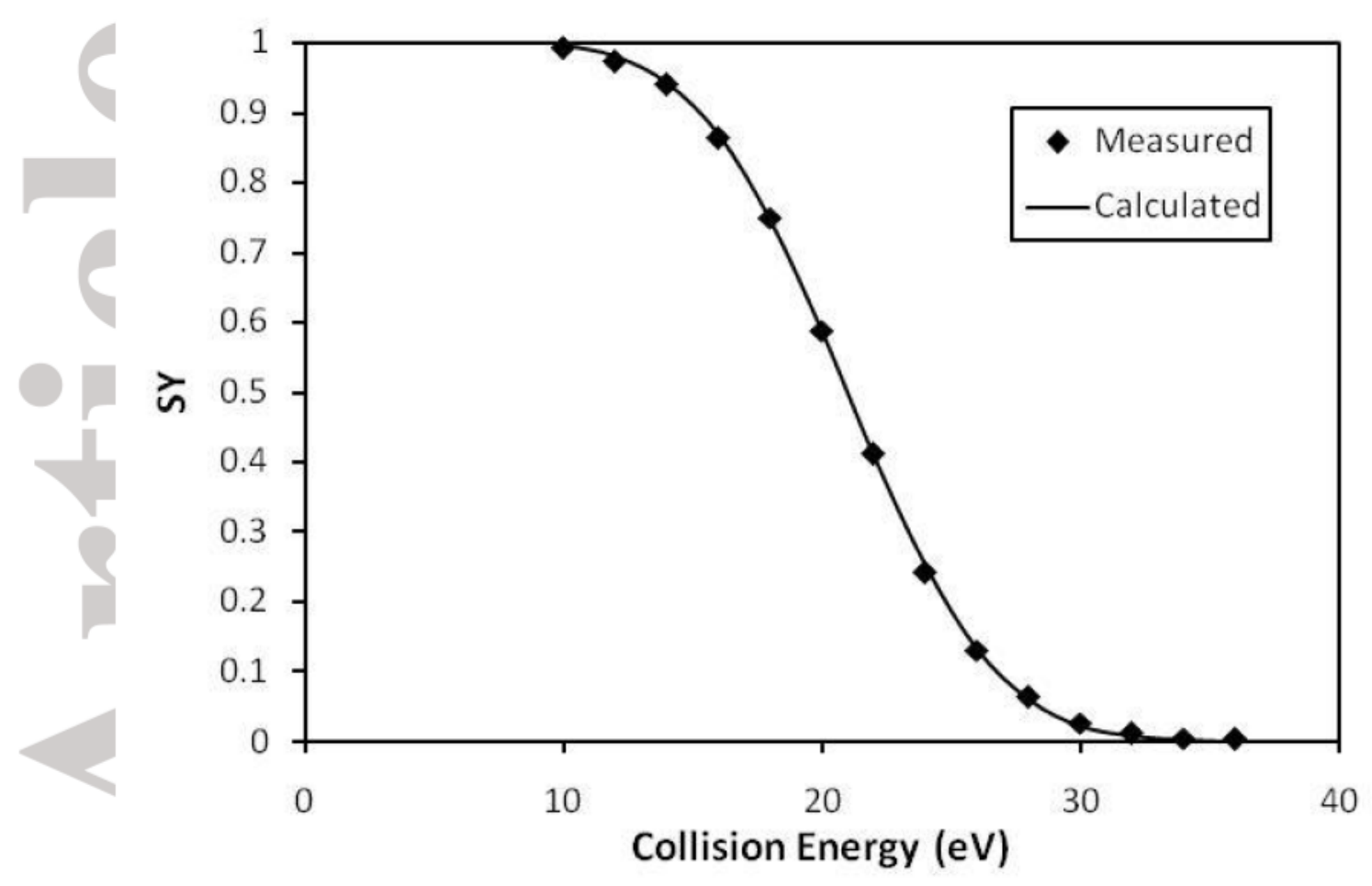

Fig. 2.

The SY values for leucine nekephalin as a function of the collision energy measured by quadrupole-time-of-flight (Q-TOF) instrument. The solid line represents the calculated curve (eq. 12) using the following parameters: $\tau=100 \mu \mathrm{s}, E_{o}=1.14, S=228, S_{\text {eff }}=46$ (calculated as $(3 \times \mathrm{N}-6) / 5$, where $\mathrm{N}$ is the number of the atoms in the precursor ion), and $E_{\text {init }}=2.05 \mathrm{eV}$ (estimated with eq. 8). The $\alpha$ and $v$ values providing the best fit were found to be 0.32 and $3.8 \times 10^{6} \mathrm{~s}^{-1}$, respectively. 
Figure 3.

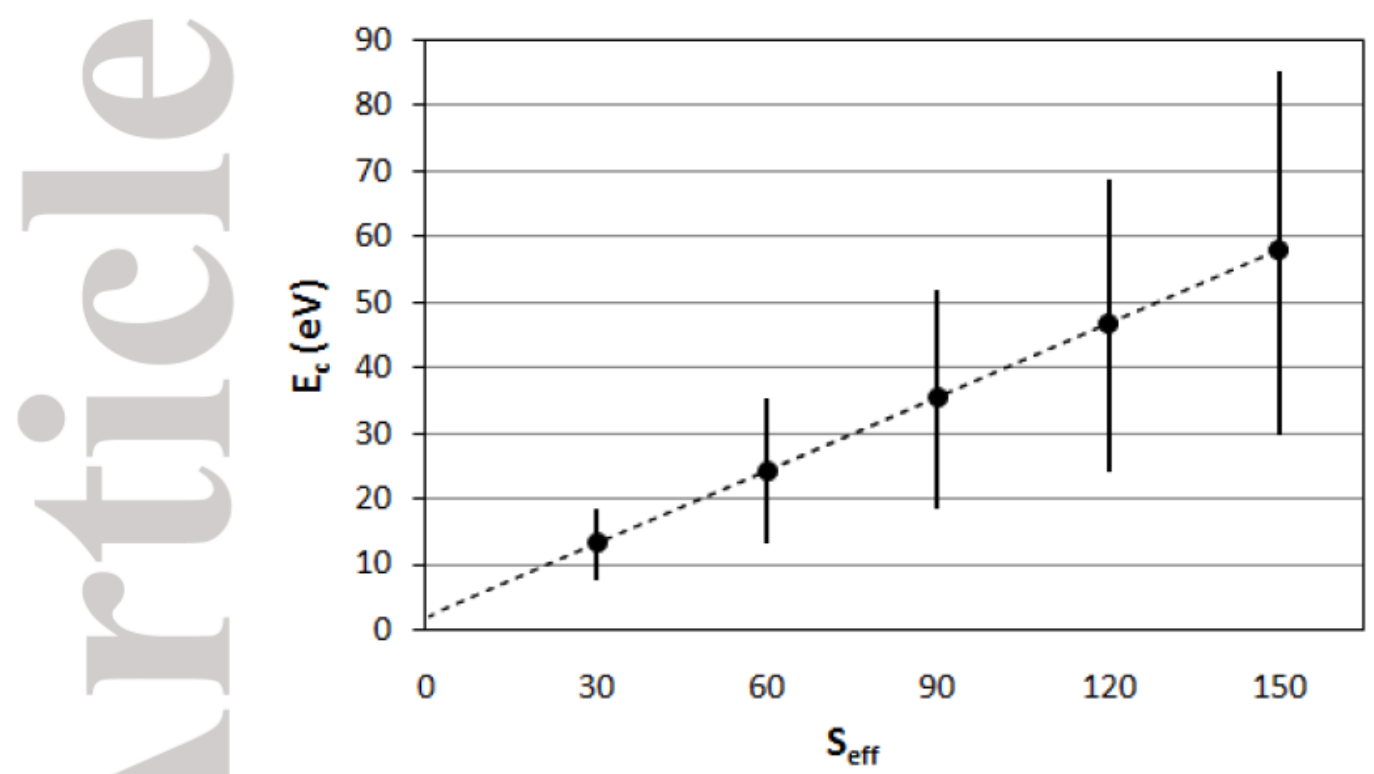

Fig. 3.

The vertical bars represent the variation of laboratory frame collision energy intervals to yield SY variable between $1-99 \%$. The black points indicate the collision energy corresponding to $50 \% \mathrm{SY}\left(\mathrm{CE}_{50}\right)$. RRK parameters were arbitrary chosen in the model calculations: $\tau=100 \mu \mathrm{s}, v=10^{7} \mathrm{~s}^{-1}, E_{o}=1.2 \mathrm{eV}$, and $\alpha=0.32$ (see eq. 12). 
Figure 4.
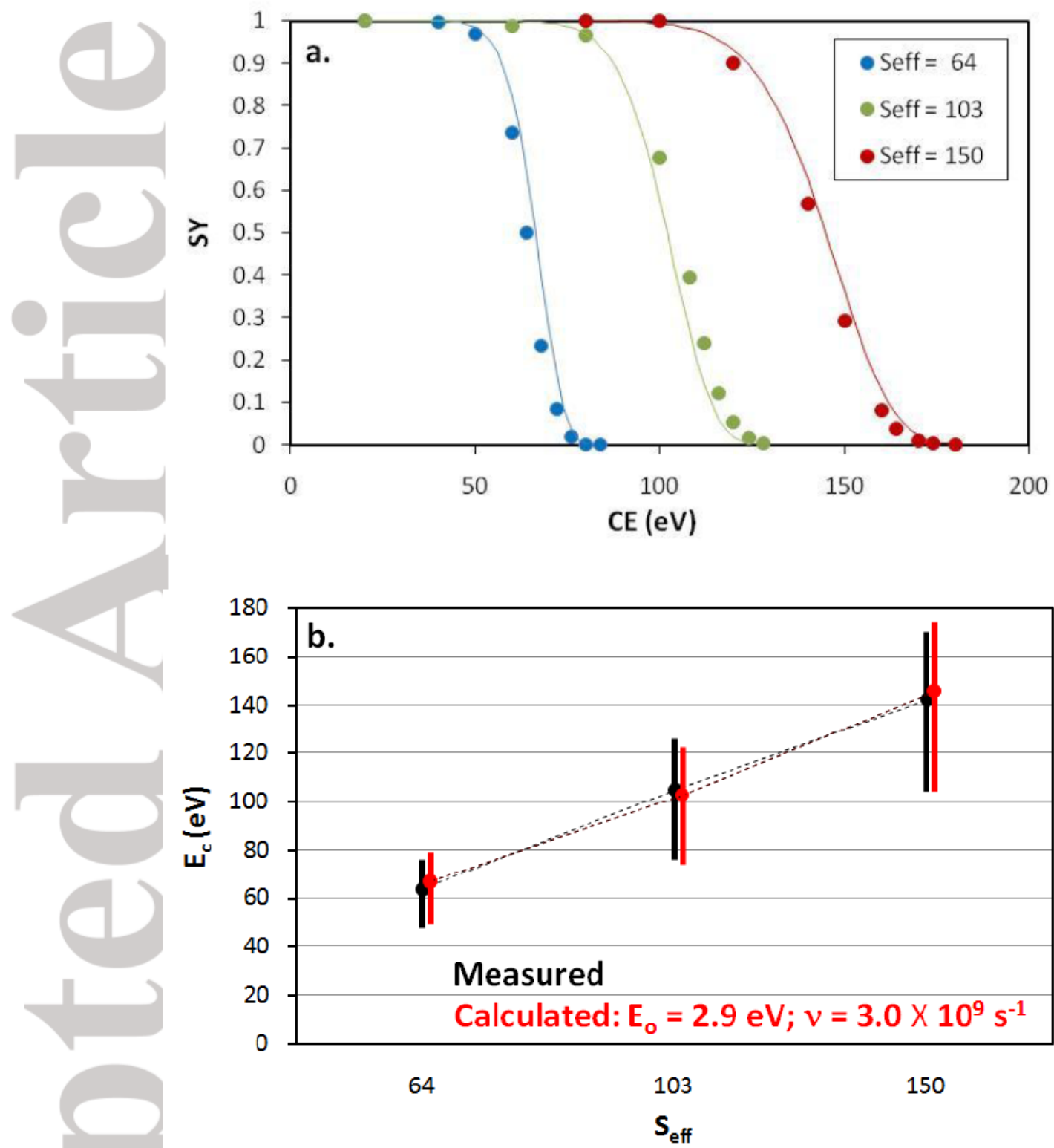

Fig. 4.

(a.) The SY values as a function of the collision energy measured by quadrupole-time-offlight (Q-TOF) instrument for doubly charged polytetrahydrofuran (PTHF) with a number of repeat units $(n)$ of 8,13 , and 19 . The solid lines represent the calculated curves using the following parameters: $\tau=100 \mu$ s and $\alpha=0.2, E_{\text {init }}$ values $2.57 \mathrm{eV}, 4.13 \mathrm{eV}$, and $6.00 \mathrm{eV}$ for PTHF with $n=8,13$, and 19 , respectively (estimated with eq. 8). The $E_{o}$ and $v$ values providing the best fit were found to be $2.9 \mathrm{eV}$ and $3.0 \times 10^{9} \mathrm{~s}^{-1}$, respectively.

(b.) Dependence of the laboratory frame collision energy intervals corresponding to the 1$99 \%$ SY range on the number of effective oscillators, black: measured, red: calculated. 
Figure 5.
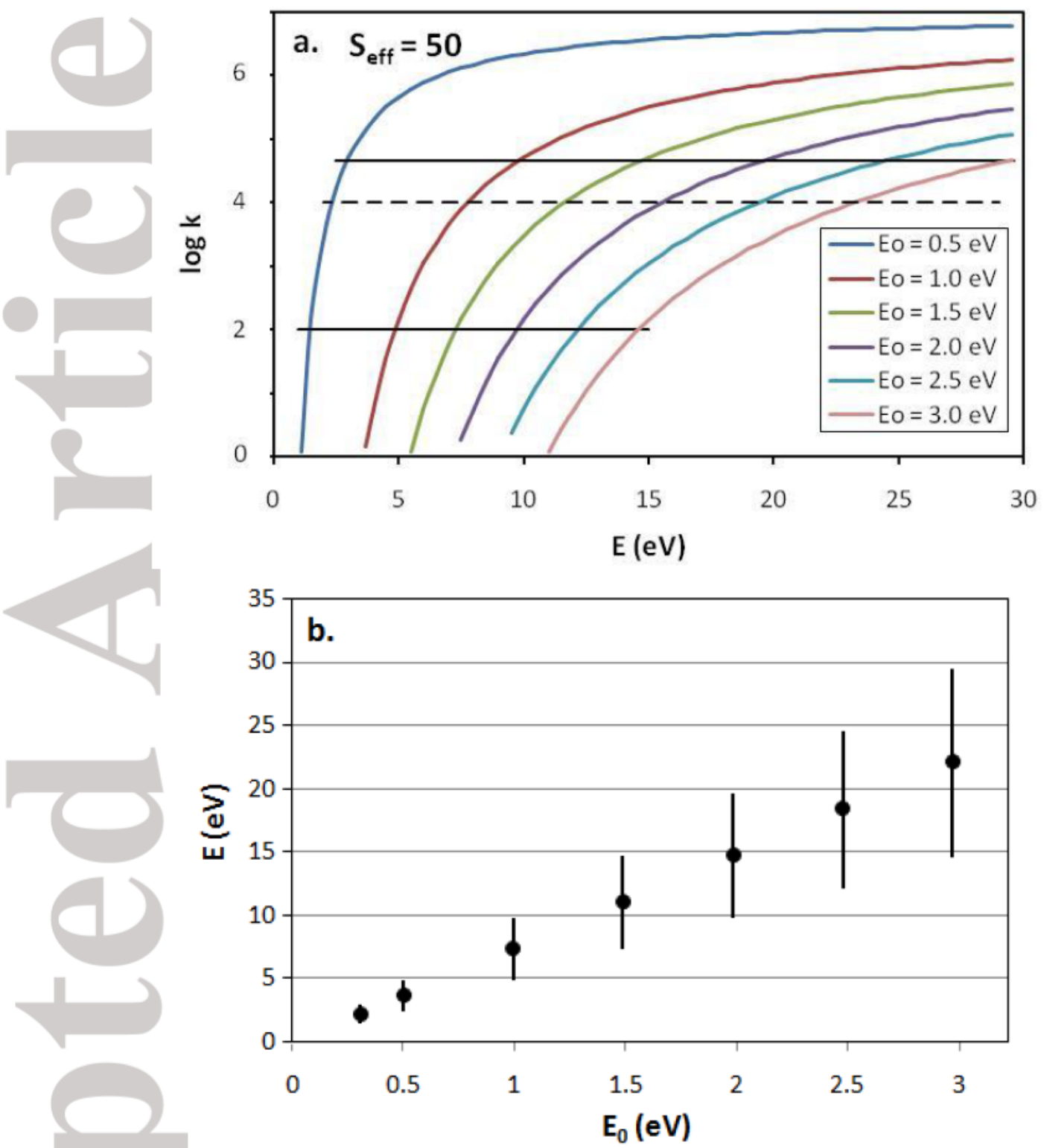


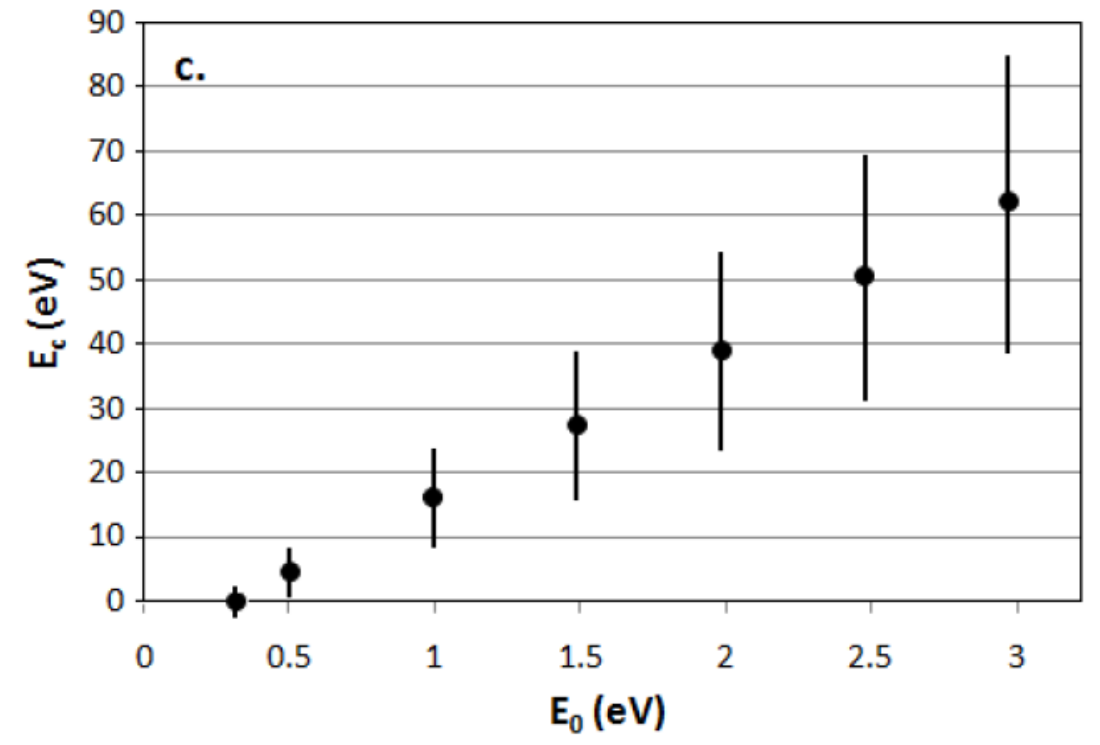

Fig. 5.

(a.) The dissociation rate coefficient $(k)$ versus internal energy $(E)$ curves calculated by the RRK equation at different critical energy values $\left(E_{o}\right)$. The horizontal lines represent the rate coefficient intervals corresponding to the $1-99 \%$ SY range. The dashed line corresponds to the $k \tau=1$ value. The vertical bars represent the variation of (b.) internal energy intervals (c.) laboratory frame collision energy intervals to yield SY variable between 1-99\%. The black points indicate the internal energy (b.) and collision energy (c.) corresponding to $50 \% \mathrm{SY}$ $\left(\mathrm{CE}_{50}\right)$. RRK parameters were arbitrary chosen in the model calculations: $\tau=100 \mu \mathrm{s}$, $v=10^{7} \mathrm{~s}^{-1}, \alpha=0.32$, and $S_{\text {eff }}=50$ (see eq. 12). 
Figure 6.

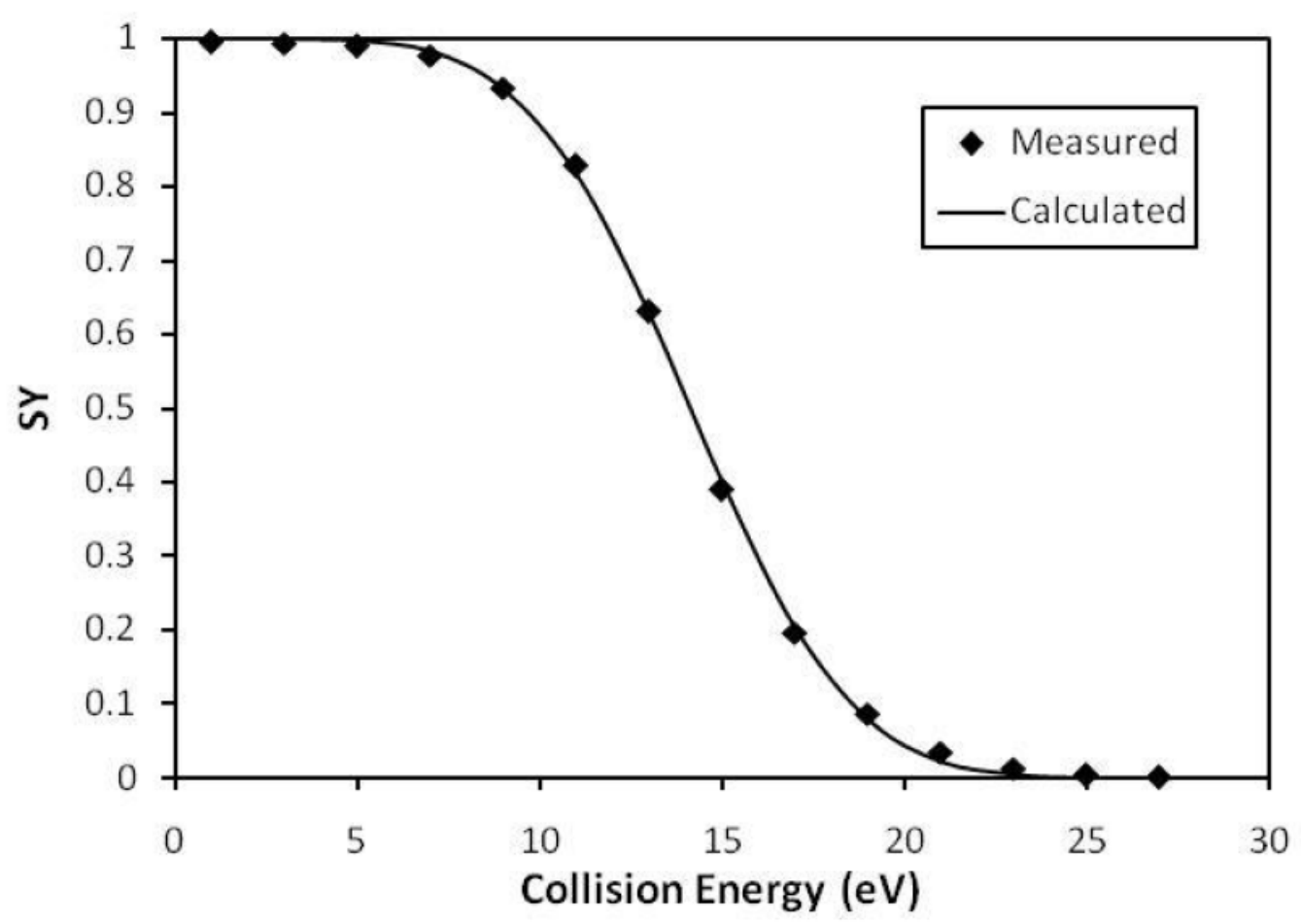

Fig. 6.

The SY values for sodiated noscapine dimer as a function of the collision energy measured by quadrupole-time-of-flight (Q-TOF) instrument. The solid line represents the calculated curve using the following parameters: $\tau=100 \mu \mathrm{s}, \alpha=0.32, S=309, S_{\text {eff }}=62$, and $E_{\text {init }}=2.78 \mathrm{eV}$. The $E_{o}$ and $v$ values providing the best fit were found to be $0.80 \mathrm{eV}$ and $9.0 \times 10^{6} \mathrm{~s}^{-1}$, respectively. 